$$
\begin{aligned}
& \text { ANL/PHY/CP_- } 90696 \\
& \text { CONF- } 9610183--3
\end{aligned}
$$

\section{NOBLE GAS SPUTTERING CALCULATIONS USING TRIM}

John P. Greene, Jennifer Nemanich and George E. Thomas

Physics Division, Argonne National Laboratory, 9700 South Cass Avenue, Argonne, IL 60439, USA

\section{Stacey L Schiel}

University of Illinois, 190 Medical Sciences Bldg.

506 S. Matthews, Urbana, IL 61801, USA
RECENED

NnV 051996

OSTI

\begin{abstract}
In conjunction with our experimental work on saddle field ion sputtering, we have attempted to apply the Monte Carlo program TRIM (TRansport of Ions in Matter) to calculate the sputter yields for a variety of noble gas sputtering applications. Comparison with experiments are shown. Information extracted from these analyses have proved useful in optimizing the experimental sputtering parameters. Calculated sputter yields obtained utilizing TRIM are presented for noble gas sputtering of a variety of materials common to nuclear target production.
\end{abstract}

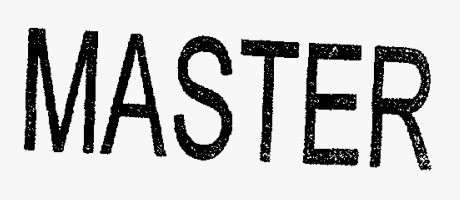

DISTRIBUTION OF THIS DOCUMENT IS UNLMITED

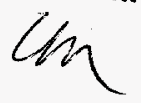




\section{DISCLAIMER}

This report was prepared as an account of work sponsored by an agency of the United States Government. Neither the United States Government nor any agency thereof, nor any of their empioyees, makes any warranty, express or implied, or assumes any legal liability or responsibility for the accuracy, completeness, or usefulness of any information, apparatus, product, or process disclosed, or represents that its use would not infringe privately owned rights. Reference herein to any specific commercial product, process, or service by trade name, trademark, manufacturer, or otherwise does not necessarily constitute or imply its endorsement, recommendation, or favoring by the United States Government or any agency thereof. The views and opinions of authors expressed herein do not necessarily state or reflect those of the United States Government or any agency thereof. 


\section{DISCLAIMER}

Portions of this document may be illegible in electronic image products. Images are produced from the best available original document. 


\section{Introduction}

In our work on the preparation of nuclear targets for heavy-ion experiments using saddle field ion sputtering of various target materials with noble gases [1], it is useful to know how the sputtering parameters vary so as to maximize the use of the expensive isotopic material employed. To this end we have attempted to calculate this information using the Monte-Carlo program TRIM (TRansport of Ions in Matter) [2]. The calculations provide direction for the physical set-up of the experimental sputtering apparatus based upon optimized values for the various sputtering parameters. We are not attempting a rigorous formalism, but instead, merely a guide as to what should work best for a particular sputtering application.

\section{The TRIM Program}

The TRIM program calculates the penetration of ions into solids and is based on the original work of J. Biersack on range algorithms [3] and J. Ziegler on stopping theory [4]. We will not attempt to present detailed source code or explanations of the various routines except those pertaining to the present sputtering calculations. The program itself is extremely simple to execute with user friendly input screens and visual displays of the interacting ions within the target. TRIM is a DOS application requiring the standard 640k memory and a math co-processor chip. In the Windows environment, we run TRIM in the background, allowing the use of the computer while the calculations are being performed. We are using TRIM Version 92.12.

When the TRIM program is invoked it will ask which type of calculation is requested. For our purposes here we wish the detailed sputtering calculations. Next it will prompt the user as to what kind of graphics (if any) to plot on the screen. The input parameters required for TRIM calculations include the incoming ion, its energy (from 10 $\mathrm{eV}$ to $2 \mathrm{GeV} / \mathrm{amu}$ ) and incident angle into the target, the composition of the target, which may contain compounds and up to three layers of differing materials and the thickness of each layer. The calculations may be carried out indefinitely, we however chose a maximum of 1000 incoming ions for all the calculations presented here. Observations of the outputted sputter yields show this to be adequate for reproducible results. Special treatment may be requested for the ions and target atoms leaving the target. Again we choose to have TRIM create a datafile of the energy and trajectory of the atoms sputtered from the target.

The program then begins the calculation with an incoming ion as it enters the target, and all kinetic phenomena related to the ions energy loss within the target. The target atom cascades are followed in detail including those which are sputtered from the surface. The display on the computer monitor consists of the chosen input parameters and plots showing the ion trajectories within the target. For increased speed these plots are normally turned off. Also shown on the screen is the number of ions the calculations have followed so far and how many are transmitted or backscattered. Finally the sputter yield 
for the ion/target combination is given as atoms/ion and the energy in eV/atom of the sputtered target atoms.

\section{Sputtering Calculations}

Sputtering is defined as the removal of near-surface atoms from the target. In this case whenever a atom from a cascade within the target has an energy greater than the surface binding energy of the target material and a trajectory which crosses the plane of the target surface. The surface binding energy of an atom to the surface of the target material is difficult to calculate, so as an estimate, the heat of sublimation for the target material is used. Although this value for the surface binding energy may be determined, in discussions with P. Sigmund [5], using the heat of sublimation proves to be a valid assumption. The sputtering calculation gives as output the sputtering yield, which is defined as mean number of target atoms sputtered per each incident ion. We are not attempting a detailed theoretical approach to the phenomena of sputtering, we will leave that to the theorists. We give here the description of the sputtering calculations supplied with the source code for TRIM.

During sputtering, only those cascades which reach back to the surface of the target give rise to the sputtering yield. Therefore it is adequate to use a thin target layer for the simulations. Although we input a thickness of $1.5 \mathrm{~mm}$ which is, on the average, the thickness of the metal foils we employed in our experiments [6], for the target "window" inputted to the program it is recommended for heavy ions, to use 40 to $50 \mathrm{~A}$ target thickness. This reduces the time spent calculating those cascades which do not reach back to the surface, and therefore do not contribute to sputtering. For light ions where backscattering may occur from deeper within the target, thicker target thicknesses of 300 to $500 \mathrm{~A}$ is adequate. The backscattered ions will cause sputtering when they exit the target surface. For the initial simulations for the noble gas ions of argon and krypton which were employed in these experiments a target thickness of $50 \mathrm{~A}$ was used.

Finally, the sputtering yield is highly dependent on the surface binding energy which, for real surfaces, changes under ion bombardment due to the surface roughness. As the sputtering involves mainly the first monolayer of the target, for heavy targets (i.e. Ni or heavier), the energy loss of the target atom traversing this monolayer approaches the surface binding energy so that changes in the surface roughness on this order may change the sputtering yield. This sensitivity of sputtering yield to the surface binding energy may be plotted during the program calculation.

More recently J.P. Biersack along with W. Eckstein have written a sputtering version of TRIM which takes into account more specific details of the sputtering process, distinguishing between primary and secondary collisions. Also treated specifically are energy and total angular distributions for ions of non-normal incidence. Although the more detailed sputtering parameters available from the TRIM.SP program [7] would be useful for the study presented here, we do not have access to this computer code at present. 


\subsection{The dependence of the sputtering yield on the energy of the noble gas sputtering beam}

As the energy of the incident ion increases from zero to some finite value where enough energy is available from the collision to allow the target atom to leave the surface, sputtering may then occur. This threshold energy depends upon the particular ion-target combination. The sputtering yield initially exhibits a quadratic dependence on the incident ion energy, and at higher energies, approaches a maximum. At even higher energies the sputtering yield actually decreases as the incident ion penetrates the target much further and collisions with the target atoms occur too deep to reach back to the surface.

As an initial first check of the sputtering calculations, a comparison study was conducted exploring the energy dependence of the noble gas ions on the sputtering yield from nickel and gold targets. For this study TRIM calculated the sputter yield from these targets using argon and krypton ions for energies from zero through $45 \mathrm{keV}$. In magnetron sputtering which employ plasmas for ionization [8], the sputtering ions impinge upon the target normal to the surface or at zero degrees for these calculations. In our experimental arrangement [9] we use focused ion beam sputtering from a saddle-field ion source positioned at 60 degrees with respect to the normal. The sputtering yields were therefore also determined for ions incident to the target at an angle of 60 degrees. In Figure 1a and $\mathrm{b}$ we plot the sputtering yields for argon and krypton ions respectively, on nickel and gold targets as a function of energy. Calculations are shown for ions incident at zero and 60 degrees. Measurements carried out in our laboratory are presented as well as comparisons from previous work on these same ion-target systems [10]. As shown in these plots, the calculations obtained using TRIM compare favorably with the experimental data.

\subsection{The dependence of the sputtering yield on the angle of the incoming sputtering beam}

The dependence of sputtering yield on the incident ion angle is well known. This is not surprising as the incident ion must impart to the target atom a velocity with a component in the outward direction from the surface in order for that particle to be sputtered. This velocity component increases at incident ion angles away from 90 degrees. The general rule for this dependence was first given by Molchanov and Tel'kovskii [11] by the relation

$$
S_{\theta}=\frac{S_{0}}{\cos \theta}
$$

where $S_{o}$ is the sputter yield at normal incidence.

Having satisfied ourselves with an initial understanding of the program results and success with the energy dependence study, we now embark upon an investigation of the dependence of the sputtering yield on the angle of the incoming ion beam. As was 
previously mentioned, we employ, in the preparation of nuclear targets, a focused ion beam sputter gun which is positioned at an angle of sixty degrees with respect to the normal to the target surface. We would like to determine the optimum angle for mounting the source so as to maximize the yield as in most instances the sputtering target is comprised of precious separated isotope. The sputtering process is dependent on the species and energy of the sputtering ion as well as the angle of incidence. There is also a variation of the sputter yield with the target material. Therefore, for our investigations into the angular dependence of the sputter yield we once again perform the calculations for combinations of argon and krypton ions incident on nickel and gold targets this time at a fixed energy of $6.8 \mathrm{keV}$. This energy was chosen as the manufacturer of the sputter source has determined that the actual energy of the ion exiting the source is $85 \%$ of the voltage potential within the source [12]. For our experimental data the source was operated at $8 \mathrm{keV}$.

We show then in Figure 2 plots of the sputter yield vs. angle of the incident ion for the four ion-target systems chosen. The sputter yield shows an increase with angle away from the normal which reaches a maximum between 65 and 70 degrees. Included in the plots along the right side $y$-axis is the number of backscattered ions as a function of the incident ion angle. Clearly as the angle of the incoming ion beam approaches 90 degrees with respect to the normal (zero degrees) a high percentage of the ions are merely backscattered and therefore cannot participate in the sputtering of target atoms. So there is some trade-off as to the positioning of the sputter source for maximum yield. In our experimental apparatus the source is mounted at an angle of 60 degrees with respect to the normal which turns out to be a good choice.

\subsection{The angular distribution of sputtered particles}

The angular distribution of the atoms sputtered from the target is also dependent upon the angle and energy of the incident ion beam. For normal incident ion beams of intermediate energy, the distribution of the sputtered particles has been shown by Andersen [13] to be cosine in nature. The distributions calculated by TRIM are assumed to possess a cylindrical symmetry perpendicular to the target surface about the point of ion impact. For non-incident ion beams this symmetry breaks down. Not withstanding we attempted to extract the angular trajectories of the target atoms sputtered from the surface using TRIM in order to get a feeling for the shape and direction of the sputtered particle distribution.

During the execution of the TRIM program one may request that a data file be constructed which contains information on the sputtered particles leaving the surface of the target. Included within this datafile is the number, final trajectory and energy of sputtered atoms. A modified version of the BASIC program TRANSMIT.BAS which is included with TRIM was written to analyze the energy and angle of the sputtered atoms and calculates a statistical distribution. The output of this program (SPUTTER.BAS) is entered into a spreadsheet for further analysis and polar plotting. A sample run using TRIM to calculate the angular distribution of nickel atoms sputtered by $1 \mathrm{keV}$ hydrogen 
ions incident on the target at an angle of 70 degrees, was performed and the resulting polar plot compared with that obtained using TRIM.SP [7]. The plots compare favorably in trajectory of the sputtered particles with the TRIM.SP results giving a somewhat wider angular distribution.

Our apparatus confines us to energies below $10 \mathrm{kV}$ and we prefer to operate at a source potential of $8 \mathrm{kV}$ which gives rise to a actual ion beam energy of $6.8 \mathrm{keV}$ as given earlier. For our purposes we will employ noble gas ions, primarily $\mathrm{Ar}$ and $\mathrm{Kr}$ incident to the surface at 60 degrees with respect to the normal. Using now these parameters, a series of calculations were performed using TRIM for Ar and $\mathrm{Kr}$ ions on a wide variety of elements common to nuclear target preparation. In Figure 3 we show polar plots of the angular distribution of sputtered $\mathrm{Ni}$ and $\mathrm{Au}$ atoms using $6.8 \mathrm{keV}$ argon and krypton beams. These distributions show a forward direction and a maximum of approximately 30 36 degrees relative to the normal to the target surface. For our experimental set-up, the collection of the sputtered material is accomplished on a target backing foil which is positioned 30 degrees relative to the normal so as to maximize the collection efficiency.

Because of this forward asymmetry, the exact shape of the distribution is difficult to visualize. The breakdown of the cylindrical symmetry which occurs for non-incident ion beams gives rise to azimuthal distributions of the sputtered particles which are not provided by TRIM. Biersack and Eckstein show an azimuthal distribution for $1 \mathrm{keV} \mathrm{H}$ atoms incident at an angle of 80 degrees on a nickel target [7]. The sideways spread in sputtered particles extends to greater than $+/-30$ degrees from the forward direction. For our experiments, a determination of this azimuthal distribution would provide a better value for the efficiency of collection of the sputtered material. For our purposes here, only a knowledge of the forward directed angle of the distribution is necessary for the optimal placement of the backing foil used to collect the sputtered particles.

\section{Experimental Results}

In our laboratory, for the preparation of the nuclear targets needed for heavy-ion accelerator based physics experiments, we employ focused ion beam sputtering as one method of production [1]. The compact sputter source contains a specially shaped central anode which produces a saddle field ionization of the gas used. For our purposes here, we will discuss operation of the sputter source using the noble gases argon and krypton. A series of depositions were made involving a number of elements used for nuclear targets. Comparisons may be made with the results calculation using TRIM by making a measurement of the ion beam current together with geometrical considerations of the target deposition.

\subsection{Description of the saddle-field ion source}

For the measurements included here, we employed a Model B21bW wide beam sputter gun [14] obtained from the VCR Group, San Francisco, CA 94080, USA. The 
source is designed to operate at levels up to $10 \mathrm{kV}$ with beam currents as high as $250 \mu \mathrm{A}$. This water-cooled sputter gun is mounted in an aluminum holder positioned at an angle of 60 degrees with respect to the normal to the sputter target surface. A photograph of the experimental apparatus is shown in Figure 4. As shown earlier this is approximately the optimal angle so as to maximize the sputter yield. The distance from the source to the sputter target is adjustable along aluminum rails which maintain this angle. The sputter source to target distance is $5 \mathrm{~cm}$.

The substrate, upon which the sputtered material is collected in order to prepare a target, consists of a suitable backing foil mounted on our standard ATLAS target frame. This substrate is positioned approximately $2 \mathrm{~cm}$ above the sputter target. The short distance increases the collection efficiency, making it possible to produce targets using small amounts of enriched isotopic source material. The backing foil is placed on a aperture mask facing the sputter target at an angle of about 30 degrees with respect to the normal. This being the position used so as to maximize the collection of the sputtered material. As previously determined for a number of elements common to target production, the sputter yield reaches a maximum at an average angle of 30 degrees with respect to the normal. For the determination of the deposition rates presented here, a small, carefully weighed thin glass disk is employed as the substrate.

\subsection{Discussion of beam current measurements}

The symmetrical geometry of the saddle field ion source produces in addition to the sputtering beam, a rear directed beam which is collected by an aluminum monitor plate. The beam exits through the $1.5 \mathrm{~mm}$ diameter rear cathode and impinges upon this electrically isolated current monitor allowing a determination of the ion beam current to be made. The measured beam current does not take into account the neutral component of the true beam intensity since the neutral particles are not detected by the current monitor. For our sputter rate measurements given here, the total beam intensity must be derived as the neutral particles incident on the target also contribute to the sputtering yield [15]. Using the published ion current and neutral component measurements supplied with this saddle field sputter gun and extrapolating to the applied source potentials used in our experiments, we estimate the neutral component of the total beam intensity to be on the order of $20 \%$. Figure shows a plot of ions + neutrals vs. accelerating voltage extrapolated to $10 \mathrm{kV}$.

The normal operating parameter for this wide beam sputter source was an accelerating potential of $8 \mathrm{kV}$. Maintaining a partial pressure for the noble gas used of 1-2 $\times 10^{-5}$ torr Ar or $\mathrm{Kr}$ within the source gives rise to a plasma current of $2 \mathrm{~mA}$. Under these conditions, the ion currents achieved varied between 40 and $50 \mu \mathrm{A}$ averaged over the one hour duration of the deposition measurements.

\subsection{Comparison with calculations}


For a comparison with the simulations obtained using TRIM, values for the experimental sputtering yield must first be derived. From our experimental depositions and adopting the erosion rate calculations of Maier [16] we derive a value for the experimental sputter yield using the equation

$$
\mathrm{Y}_{\mathrm{exp}}=\frac{\mathrm{R}}{37.32 * \mathrm{M}^{*} \mathrm{I}_{\mathrm{t}}}
$$

where $\mathrm{R}$ is the erosion rate of the material and $\mathrm{M}$ is the mass of the species being sputtered. The total beam intensity, $I_{t}$ is given in $\mathrm{mA}$ and is derived from the measured ion beam current and modified to include the neutral component.

In order to derive the erosion rate $\mathrm{R}$, we use the measured average film thickness condensed per hour, $T$ which is given in units of $\mu \mathrm{g} / \mathrm{cm}^{2} \mathrm{~h}$.

$$
R=\frac{\pi D^{2}}{4 \eta} T
$$

The diameter of the measured deposit, $\mathrm{D}$ is equal to $0.953 \mathrm{~cm}$ in our experiments. Maier assumes a cosine distribution for the sputtered atoms, with the efficiency given as

$$
\eta=1-\frac{h^{2}}{\left(h^{2}+D^{2} / 4\right)}
$$

where for our geometry $\mathrm{h}$ is equal to $2 \mathrm{~cm}$. In the experiments conducted by Maier $\eta=12.3 \%$ and for our geometry this collection efficiency would calculate to $5.3 \%$, again, assuming a cosine distribution. As we have shown, the sputtered particle distribution exhibits a forward direction due to the non-incident ion beam. This nonincidence of the ion beam brings into question the assumption of a cosine distribution for the sputtered particles. This forward asymmetry also produces a azimuthal distribution of sputtered atoms. A more exact determination of the collection efficiency for our experiments requires knowledge of the angular distribution of the sputtered particles including the azimuthal component. We choose an efficiency of $10 \%$ as a reasonable value for $\eta$ until a detailed examination of the total angular distribution can be determined.

Given now the experimentally determined sputter yields for a number of elements used in target production and obtained using sputtering beams of the noble gases argon and krypton, we show in Tables I and II the comparison with TRIM calculations. For the calculated sputter yields, the energy of the sputtering beam was $6.8 \mathrm{keV}$ and the incident angle 60 degrees. The simulations were done within a surface target layer $300 \mathrm{~A}$ thick which takes into account the oblique incidence of the incoming ion beam. The TRIM calculations were carried out for 1000 incident ions.

We find close agreement for many of the ion-target combinations shown. In some instances it appears that the calculated yield is much higher especially in the case for argon 
on zinc. We must once again stress that modeling done within TRIM doesn't take into account the surface erosion of the sputter target. For the zinc we attribute this to its heat of sublimation which is the value TRIM is using as an approximation to the surface binding energy.

\section{Conclusion}

In conclusion we find that a better understanding of the sputtering process in the production of nuclear targets has been achieved using the simulations provided by TRIM. Optimization of the experimental parameters used in our laboratory will lead to higher sputter yields and efficiencies regarding the use of enriched separated isotope. The yields derived for noble gas sputtering using TRIM compare favorably with the experimental data. Detailed calculations on specific systems may be further explored using this computer program.

\section{Acknowledgments}

The authors would like to thank Dr. Walter Henning, the Physics Division Director, and Dr. Irshad Ahmad, the Target Facility Group Leader, for their continuing encouragement and support of these efforts. We would also like to thank Randall Gasa of Ohio University for the angular distribution calculations of the selected elements. This work is supported by the U.S. Department of Energy, Nuclear Physics Division, under Contract No. W-31-109-Eng-38. 


\section{References}

[1] G.E. Thomas, Proc. Workshop of the International Nuclear Target Development Society, 1983 ANL/PHY-84-2, p. 249.

[2] "The Stopping and Range of Ions in Solids" by J.F. Ziegler, J.P. Biersack and U. Littmark, Pergamon Press, New York, NY, USA (1985)

[3] J. Biersack and L. Haggmark, Nucl. Instr. and Meth., 174, (1980), 257.

[4] "The Stopping and Range of Ions in Matter" by J.F. Ziegler, vol. 2-6, Pergamon Press, New York, NY USA (1977-1980)

[5] P. Sigmund, priv. comm.

[6] John P. Greene, George E. Thomas and Stacey Schiel, presented at this Conference.

[7] J.P. Biersack and W. Eckstein, Appl. Phys. A 34, (1984), 73-94

[8] G. Sletten and P. Knudsen, Nucl. Instr. and Meth. 102 (1972), 459-463

[9] G.E. Thomas and J.P. Greene, Nucl Instr. and Meth. A334 (1993) 132-136

[10] "Ion Bombardment of Solids" by G. Carter and J.S. Colligon, Chapter 7, American Elsevier Publishing Company, Inc., New York, NY USA

[11] V.A. Molchanov and V.G. Tel'Kovskii, Bull. Acad. Sci. USSR Phys. Ser. 26, (1963), 1381.

[12] "Ion Beam Micro-Sputtering of Thin Films", Ion Tech Ltd. 2 Park Street, Teddington, Middlesex, TW11 OLT, England

[13] H.H. Andersen, Nucl. Instr. and Meth. in Phys. Res. B33, (1988), 466-473

[14] B21 WIDE BEAM SADDLE FIELD ION SOURCE, Information Sheet C, Ion Tech Ltd. 2 Park Street, Teddington, Middlesex, TW11 OLT, England

[15] B11 FINE BEAM SADDLE FIELD ION SOURCE, Information Sheet B, Ion Tech Ltd. 2 Park Street, Teddington, Middlesex, TW11 OLT, England

[16] H.J. Maier, Nucl Instr. and Meth. A303 (1991), 172-181. 


\section{Tables}

Table I - Table Experimental Sputter Yields using $6.8 \mathrm{keV}$ argon ions incident at an angle of 60 degrees relative to the normal to the surface of the sputter targets as compared to Calculated Sputter Yields using TRIM. The targets were comprised of elements common to the preparation of nuclear targets

Table I I - Table Experimental Sputter Yields using $6.8 \mathrm{keV}$ krypton ions incident at an angle of 60 degrees relative to the normal to the surface of the sputter targets as compared to Calculated Sputter Yields using TRIM. The targets were comprised of elements common to the preparation of nuclear targets

\section{Figure Captions}

Figure 1a. Plots of the calculated Sputter Yield vs. Energy of argon ions at zero and 60 degrees using TRIM. Experimental data points from the present work and comparisons with previous work are also plotted.

Figure 1b. Plots of the calculated Sputter Yield vs. Energy of krypton ions at zero and 60 degrees using TRIM. Experimental data points from the present work and comparisons with previous work are also plotted.

Figure 2a. Plots of the calculated Sputter Yield vs. Angle of the $6.8 \mathrm{keV}$ argon ions incident on nickel and gold sputtering targets using TRIM. Also included is the calculated Backscatter Ions vs. Angle. The curves have been fitted and smoothed.

Figure 2b. Plots of the calculated Sputter Yield vs. Angle of the $6.8 \mathrm{keV}$ krypton ions incident on nickel and gold sputtering targets using TRIM. Also included is the calculated Backscatter Ions vs. Angle. The curves have been fitted and smoothed.

Figure 3a. Polar plots of the Angular Distribution of the nickel and gold sputtered atoms by $6.8 \mathrm{keV}$ argon ions incident at 60 degrees relative to the normal to the surface.

Figure 3b. Polar plots of the Angular Distribution of the nickel and gold sputtered atoms by $6.8 \mathrm{keV}$ krypton ions incident at 60 degrees relative to the normal to the surface.

Figure 4. Photograph showing the B21bW Wide Beam Saddle Field Ion Source and experimental sputtering set-up. 
Table I

\section{ARGON}
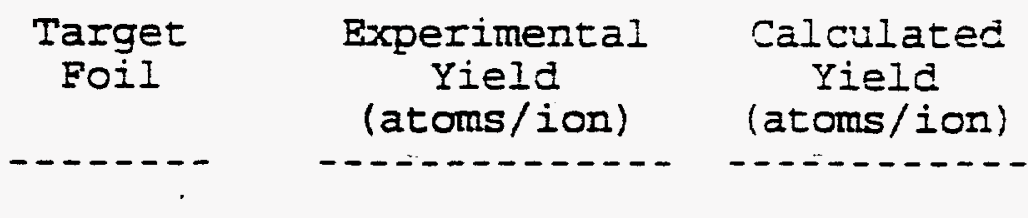

$\mathrm{C}$
$\mathrm{Mg}$
$27 \mathrm{Al}$
$\mathrm{Ti}$
$\mathrm{Cr}$
$\mathrm{Fe}$
$\mathrm{Ni}$
$\mathrm{Cu}$
$\mathrm{Zn}$
$\mathrm{Mo}$
$\mathrm{Ag}$
$\mathrm{Sn}$
$\mathrm{W}$
$\mathrm{Pt}$
$197_{\mathrm{Au}}$
$\mathrm{Pb}$
$209_{\mathrm{Bi}}$

1.39
9.84
6.83
4.61
11.98
6.42
15.23
15.42
22.04
5.64
17.00
12.54
3.99
7.99
11.56
9.86
10.62

1.87

8.79

5.99

5.19

11.09

11.46

13.85

18.95

43.59

6.82

19.51

$-10.02$

6.00

8.72

13.15

10.82

8.65 


\section{KRYPIFON}

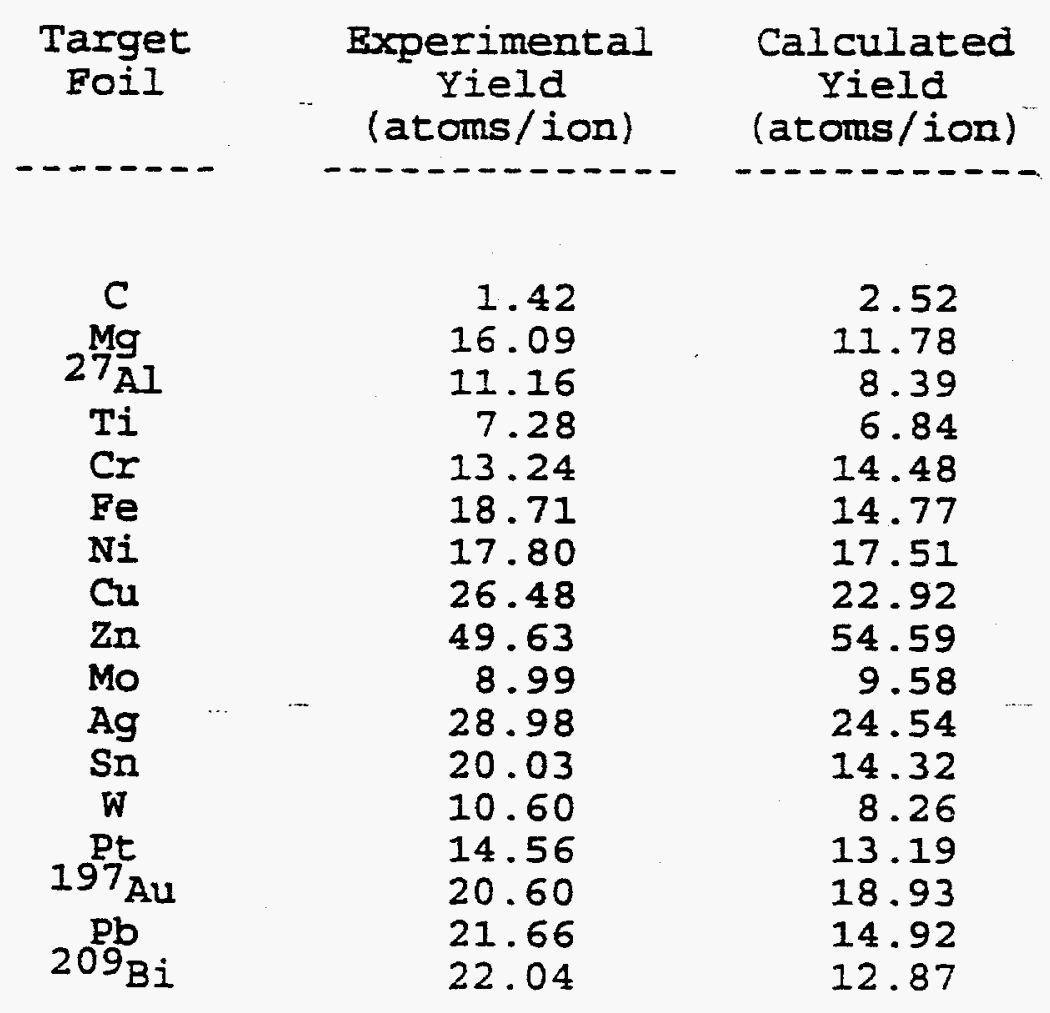



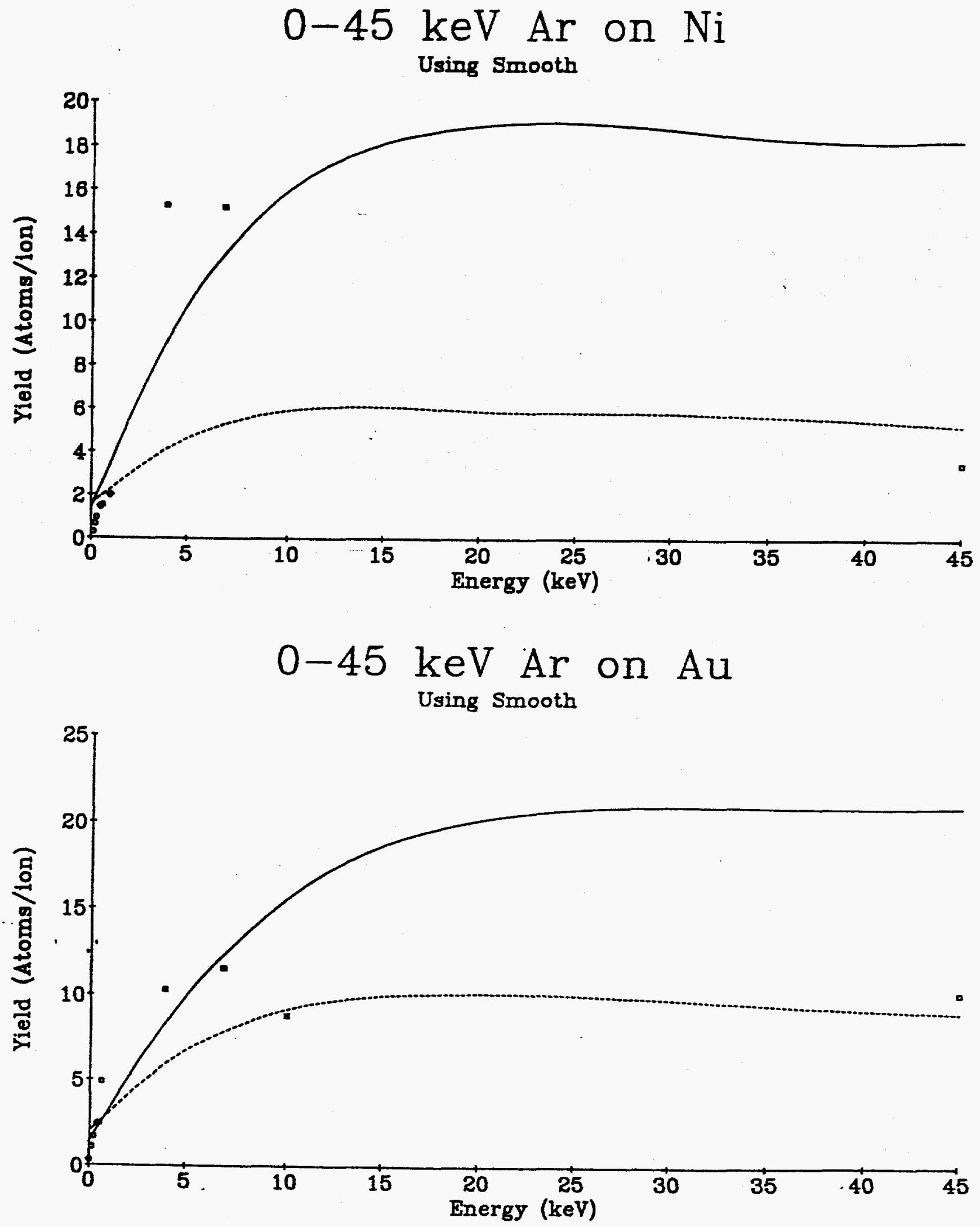

- Present Work -....-TRM Calculation at 0 degrees TRIM Calculation at 90 degrees

O N. Laegried and G.K. Wehner, J. Appl. Phys. 32, (1961) 365

O. Almen and G. Bruce, Nucl. Instr. and Meth. 11, (1961) 257

* O. Almen and G. Bruce, Trans. 8th National Vacuum Symp., Washington, Pergamon Press, Inc., New York, 1, (1962) 245

() G.K. Wehner, General Mills Report No. 2309 (1962)

D. McKeown and A.Y. Cabezas. Ann. Revort Space Sci. Lab.. General Dvnamics/Astronautics (1962) 

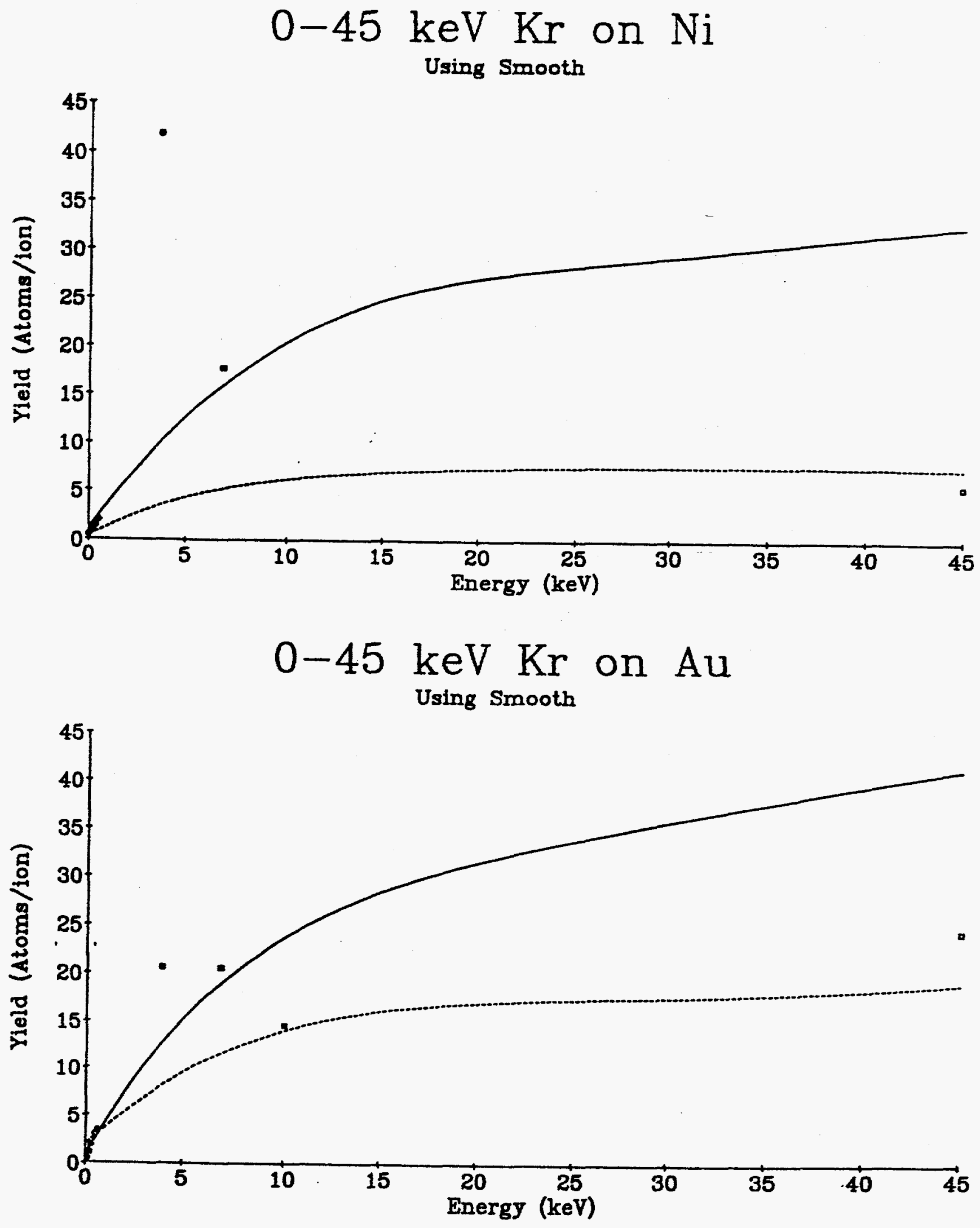

- Present Work -...--TRIM Calculation at 0 degrees TRMM Calculation at 90 degrees

D. Rosenburg and G.K. Wehner, J. Appl. Phys. 33, (1962) 1842

$\square \quad$ O. Almen and G. Bruce, Nucl. Instr. and Meth. 11, (1961) 257

* O. Almen and G. Bruce, Trans. 8th National Vacuum Symp., Washington, Pergamon Press, Inc., New York, 1, (1962) 245

C) G.K. Wehner, General Mills Report No. 2309 (1962)

- D. McKeown and A.Y. Cabezas, Ann. Report Space Sci. Lab., General Dynamics/Astronautics (1962) 


\section{$6.8 \mathrm{keV} \mathrm{Ar}$ on $\mathrm{Ni}$ \\ Using Smooth}

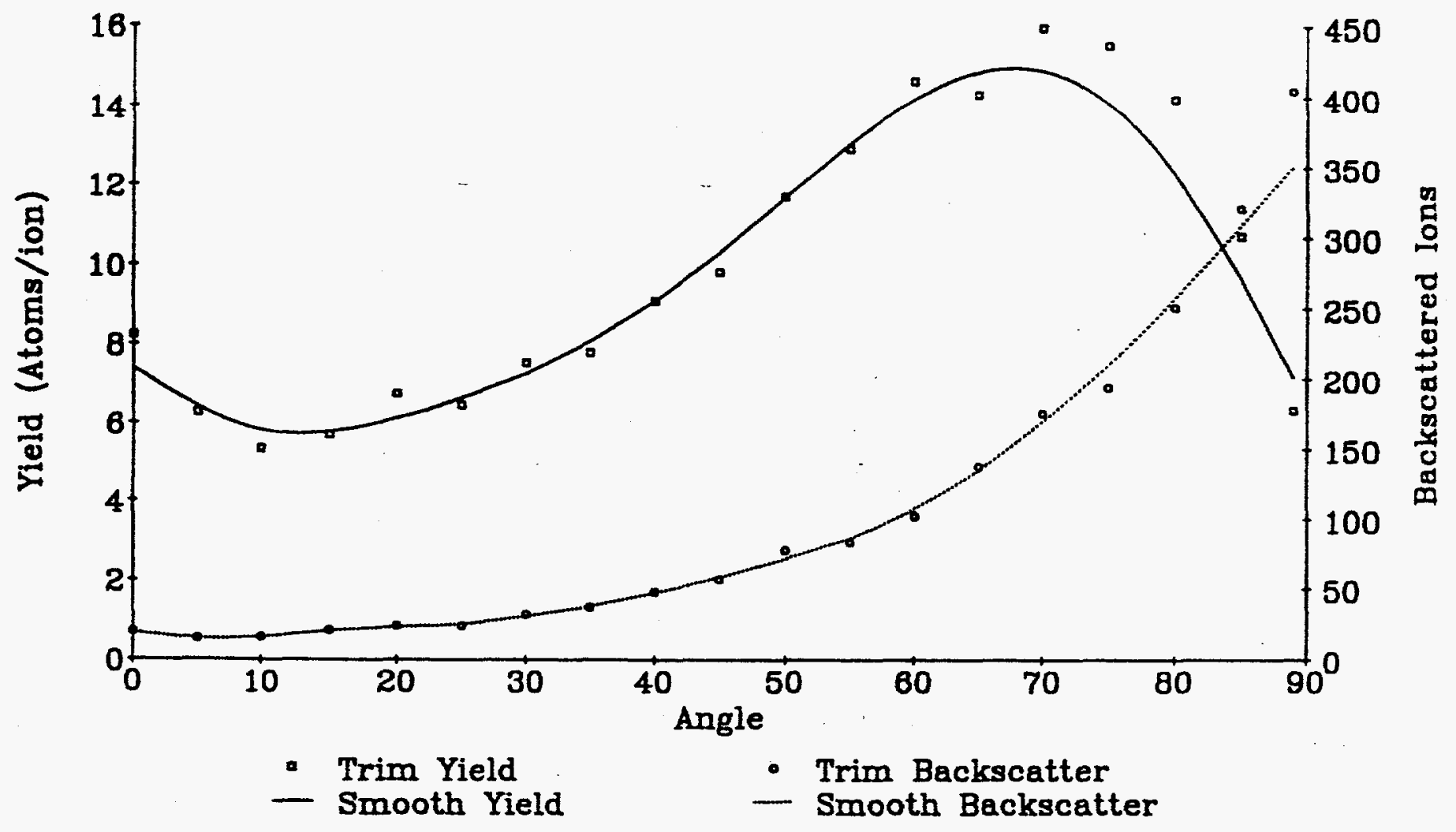

\section{$6.8 \mathrm{keV}$ Ar on $\mathrm{Au}$}

\section{Using Smooth}

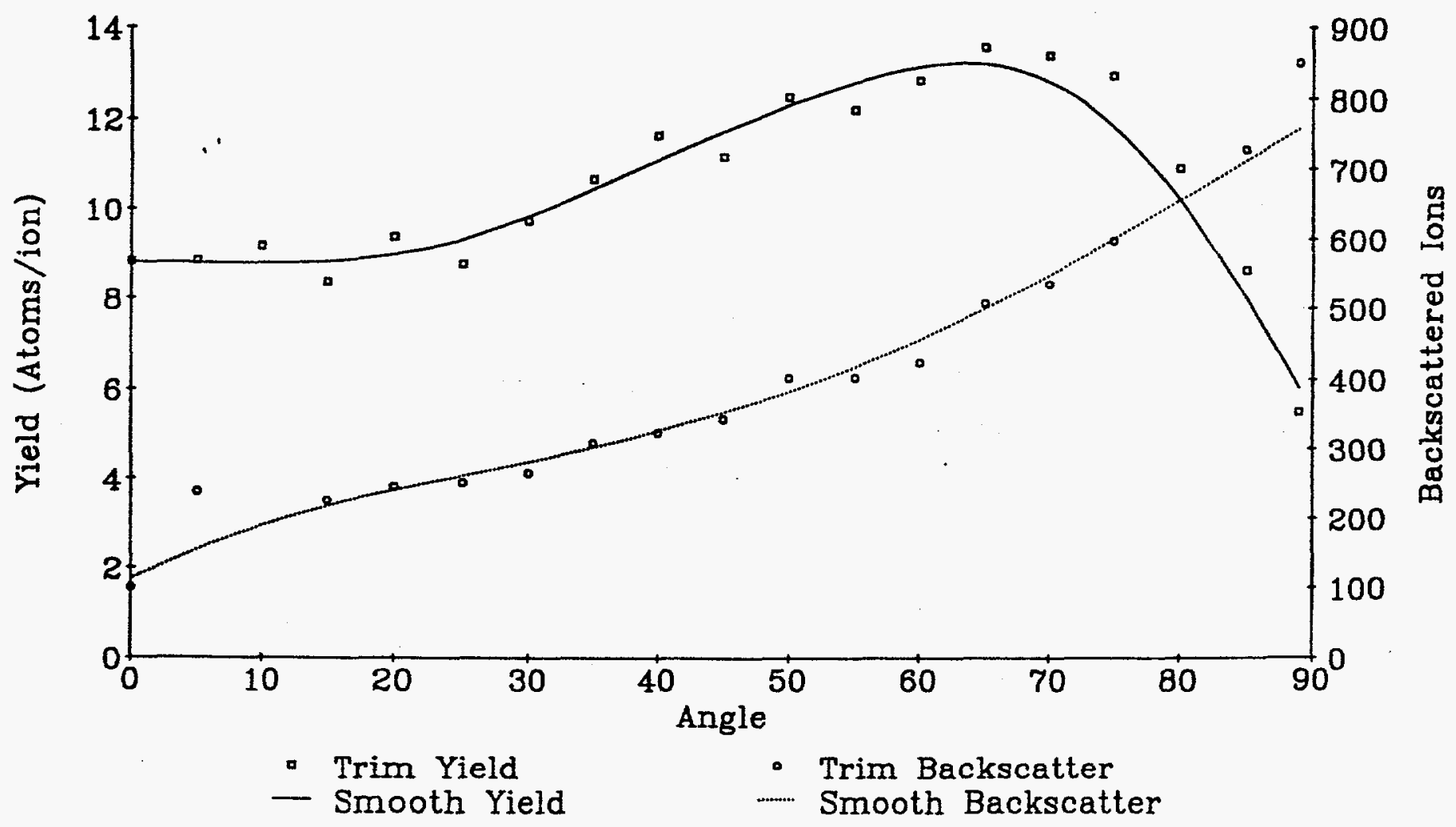

Figure 2a 


\section{$6.8 \mathrm{keV} \mathrm{Kr}$ on $\mathrm{Ni}$ \\ Using Smooth}

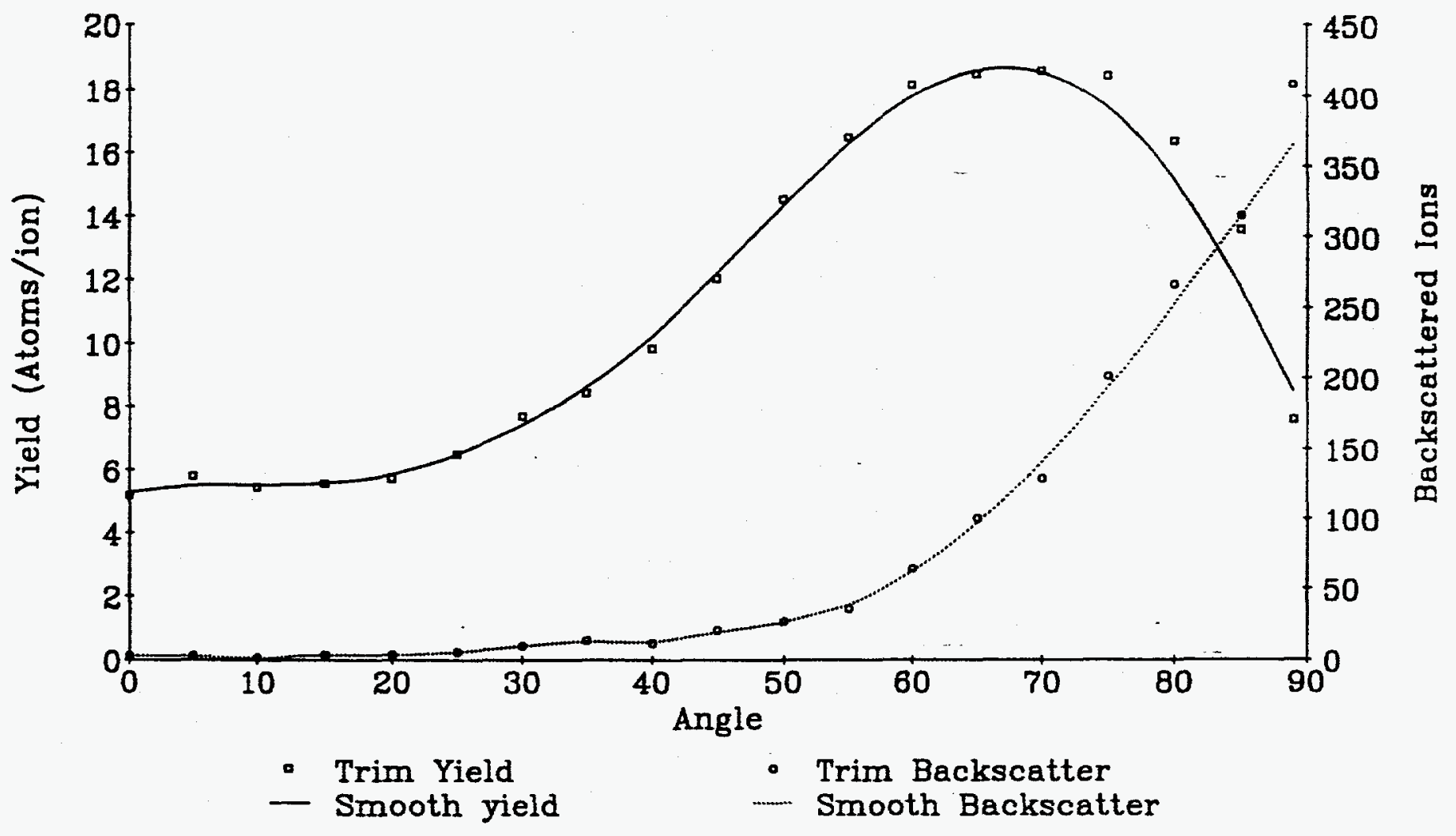

$6.8 \mathrm{keV} \mathrm{Kr}$ on $\mathrm{Au}$

Using Smooth and Least Squares

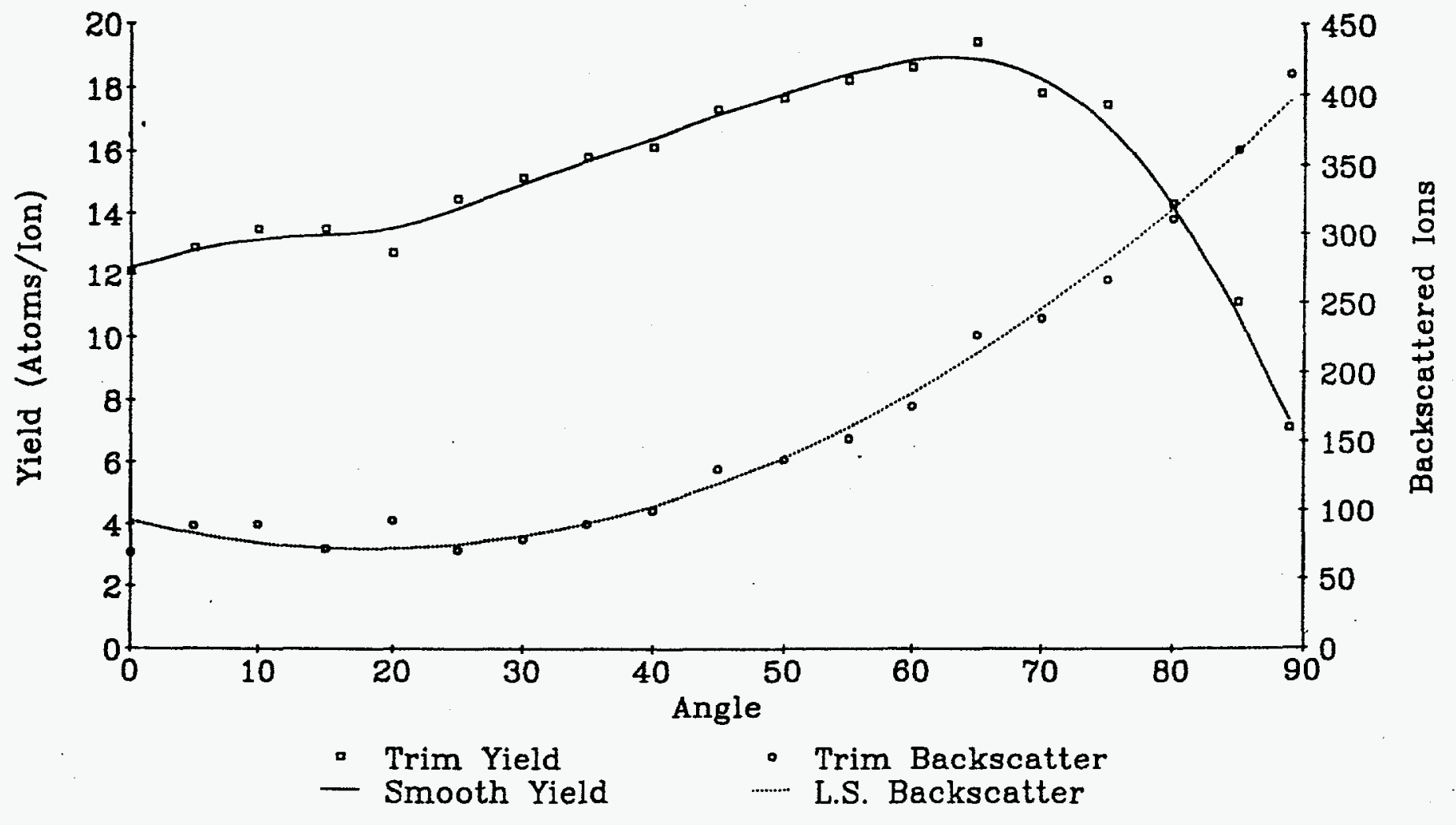

Figure $2 b$ 
$6.8 \mathrm{keV}$ Ar Sputtering @ 60

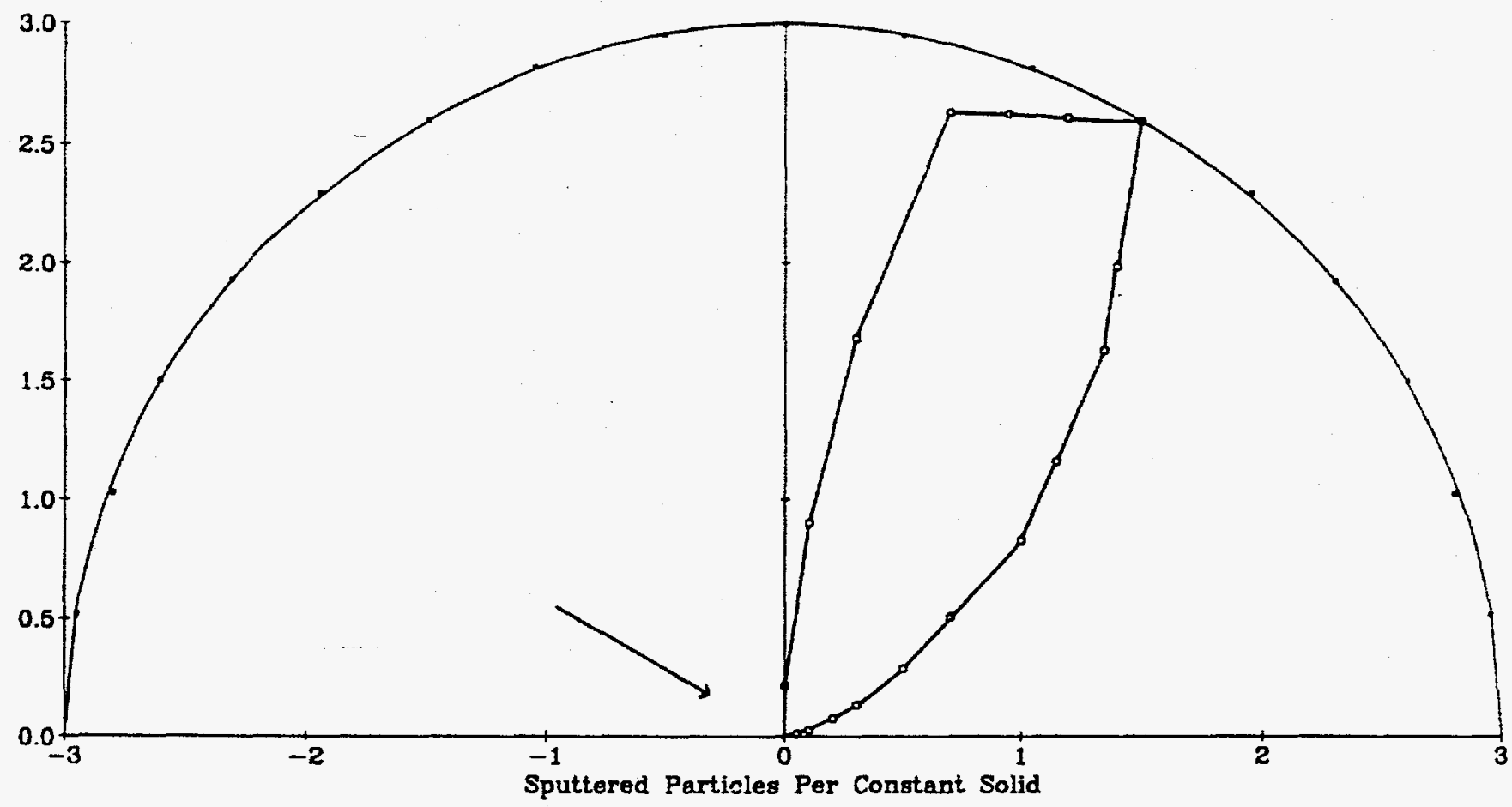

$6.8 \mathrm{keV}$ Ar Sputtering (9) 60 Target $=\mathrm{Au}$

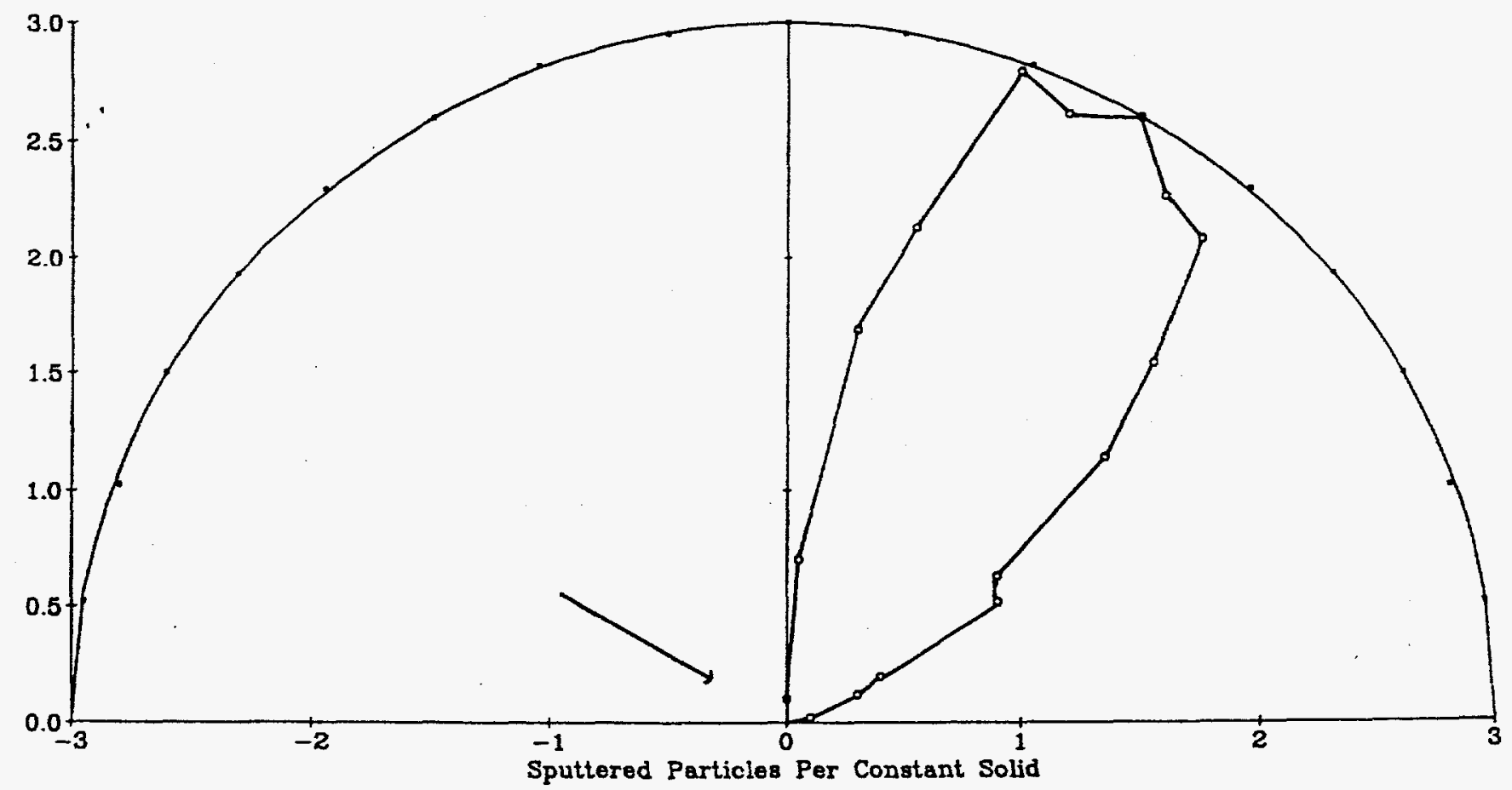

Figure $3 a$ 


\section{$6.8 \mathrm{keV} \mathrm{Kr}$ Sputtering (9)60 Target $=\mathrm{Ni}$}

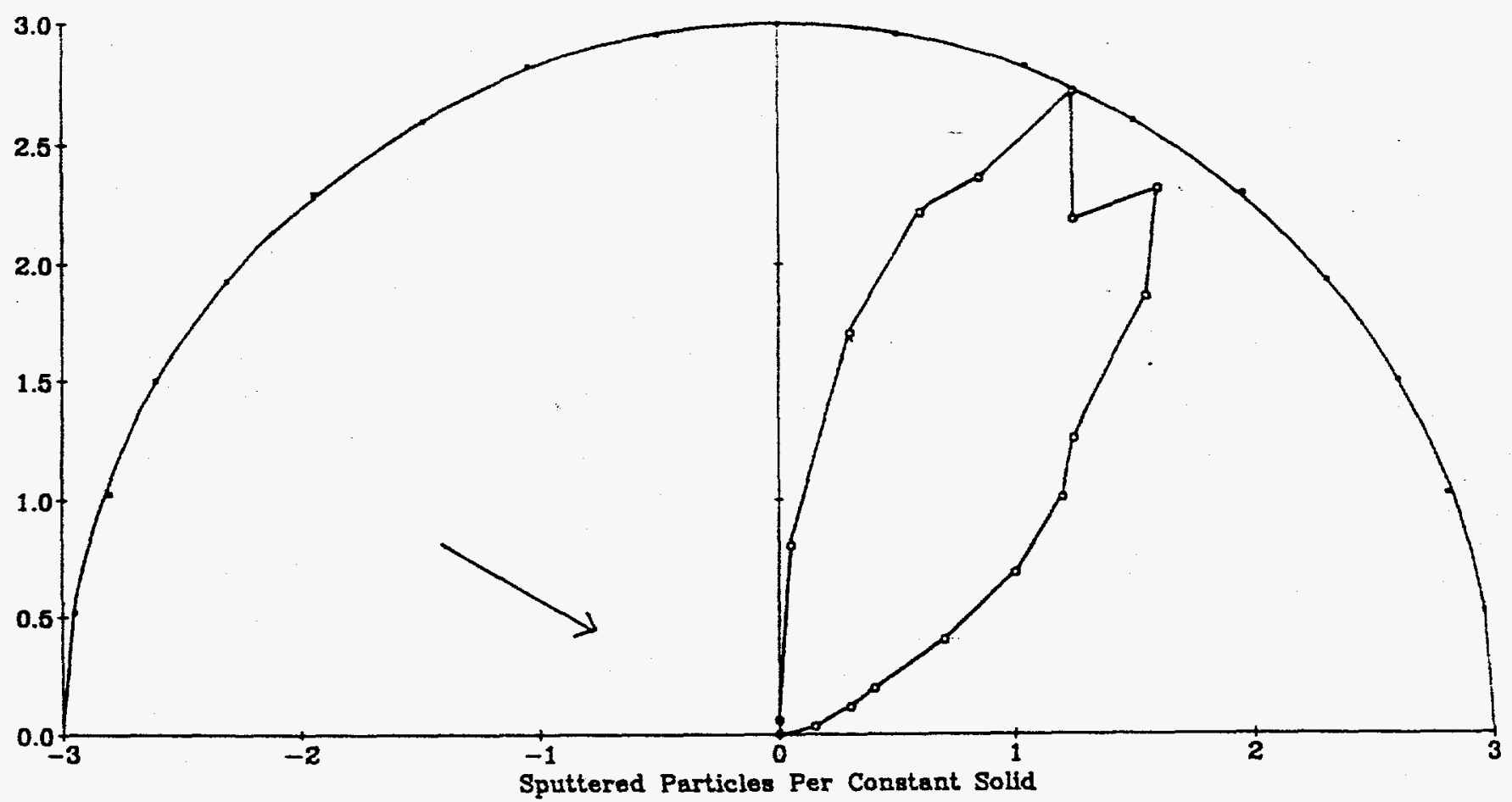

$6.8 \mathrm{keV} \mathrm{Kr}$ Sputtering (9) 60

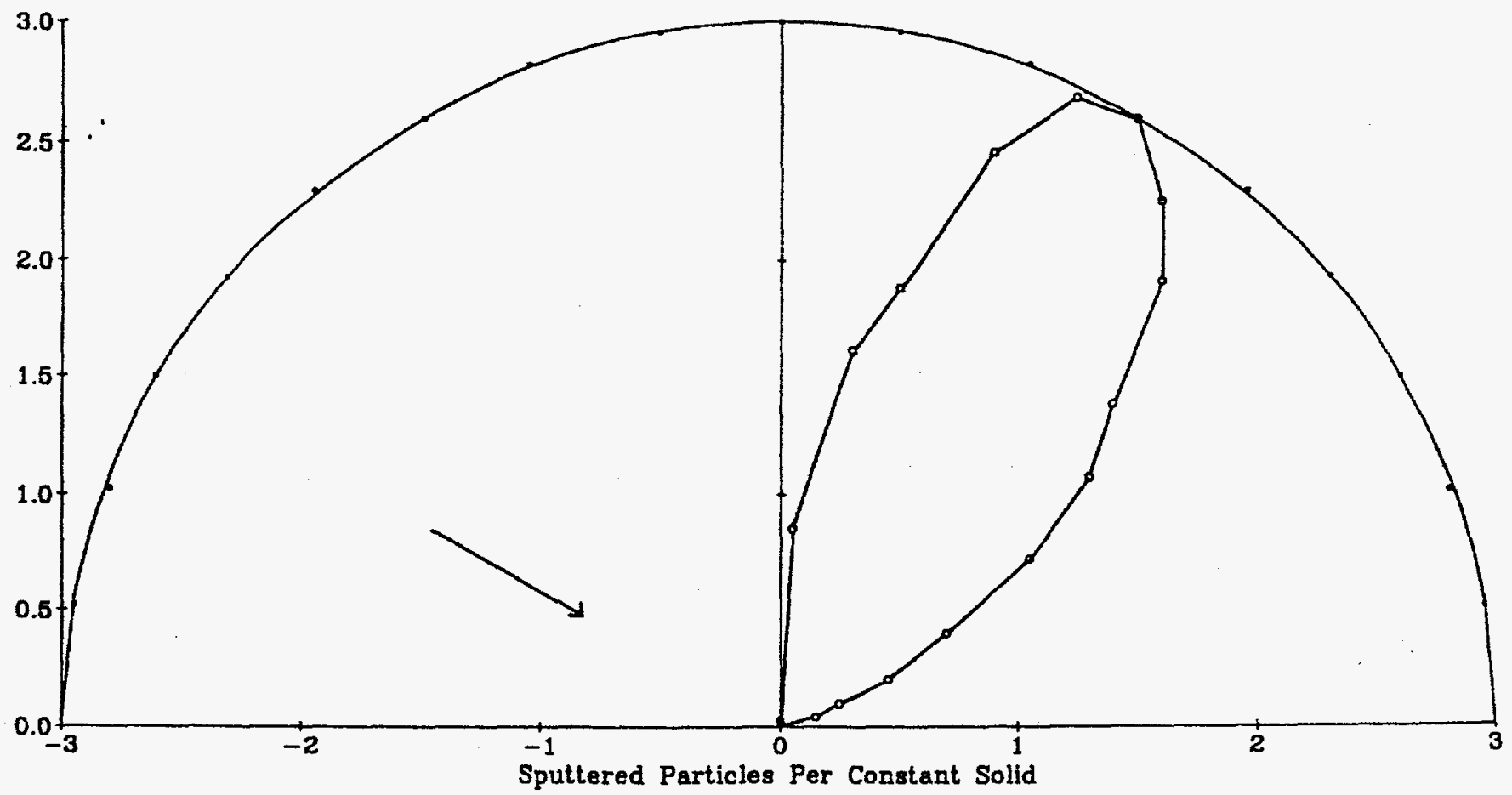

Figure $3 b$ 


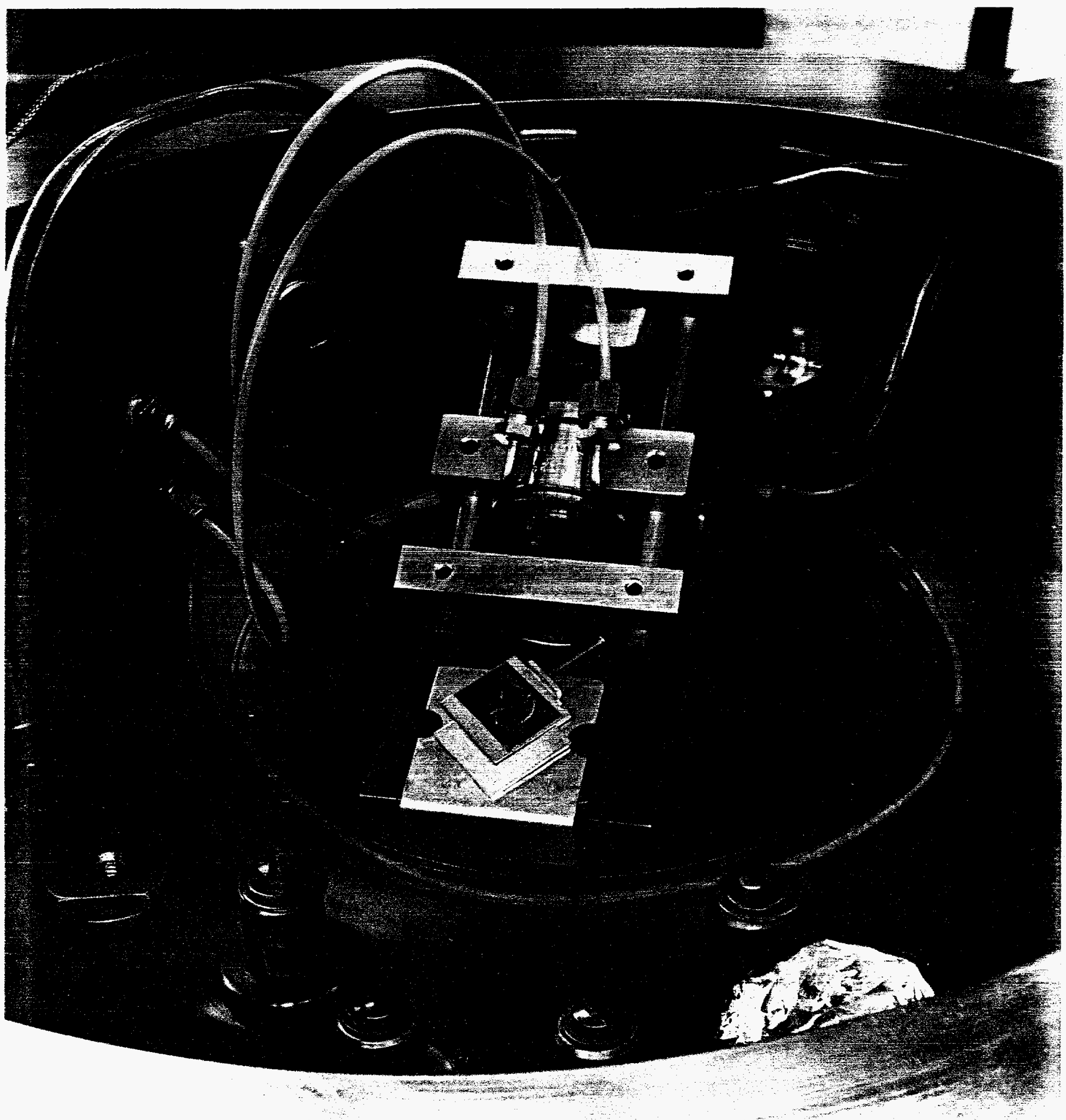

Figure 4 